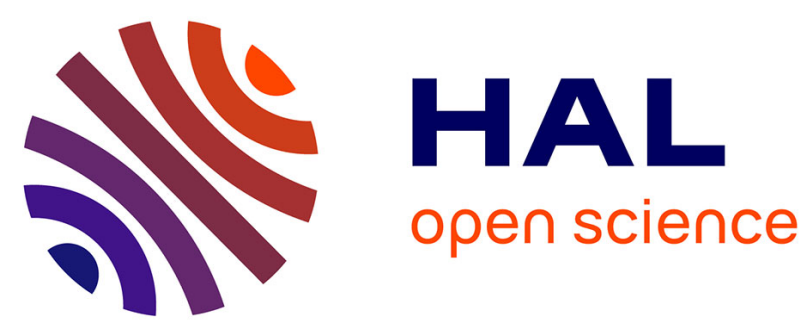

\title{
L'AGGLOMÉRATION À FLUX TENDUS.LA POLITIQUE DE SÉCURITÉ CIVILE FRANÇAISE AU SECOURS DES PANNES URBAINES
}

Mathilde Gralepois

\section{- To cite this version:}

Mathilde Gralepois. L'AGGLOMÉRATION À FLUX TENDUS.LA POLITIQUE DE SÉCURITÉ CIVILE FRANÇAISE AU SECOURS DES PANNES URBAINES. Flux - Cahiers scientifiques internationaux Réseaux et territoires, 2010, 81, pp.57-66. halshs-01259774

\section{HAL Id: halshs-01259774 \\ https://shs.hal.science/halshs-01259774}

Submitted on 20 Jan 2016

HAL is a multi-disciplinary open access archive for the deposit and dissemination of scientific research documents, whether they are published or not. The documents may come from teaching and research institutions in France or abroad, or from public or private research centers.
L'archive ouverte pluridisciplinaire HAL, est destinée au dépôt et à la diffusion de documents scientifiques de niveau recherche, publiés ou non, émanant des établissements d'enseignement et de recherche français ou étrangers, des laboratoires publics ou privés. 


\section{L'AGGLOMÉRATION À FLUX TENDUS. LA POLITIQUE DE SÉCURITÉ CIVILE FRANÇAISE AU SECOURS DES PANNES URBAINES}

Mathilde Gralepois

Métropolis | « Flux »

2010/3 n 81 I pages 57 à 66

ISSN 1154-2721

Article disponible en ligne à l'adresse :

http://www .cairn.info/revue-flux-2010-3-page-57.htm

\section{!Pour citer cet article :}

Mathilde Gralepois, «L'agglomération à flux tendus. La politique de sécurité civile française au secours des pannes urbaines », Flux 2010/3 (n 81), p. 57-66.

Distribution électronique Cairn.info pour Métropolis.

(C) Métropolis. Tous droits réservés pour tous pays.

La reproduction ou représentation de cet article, notamment par photocopie, n'est autorisée que dans les limites des conditions générales d'utilisation du site ou, le cas échéant, des conditions générales de la licence souscrite par votre établissement. Toute autre reproduction ou représentation, en tout ou partie, sous quelque forme et de quelque manière que ce soit, est interdite sauf accord préalable et écrit de l'éditeur, en dehors des cas prévus par la législation en vigueur en France. Il est précisé que son stockage dans une base de données est également interdit. 


\title{
L'agglomération à flux tendus. La politique de sécurité civile française au secours des pannes urbaines
}

\author{
Mathilde Gralepois
}

\section{INTRODUCTION}

$\mathrm{E}^{\mathrm{n}}$ n 2004, la politique française de sécurité civile énonce expli- citement ses orientations dans une nouvelle loi dite de « modernisation » (1). Le premier article rappelle que « la sécurité civile a pour objet la prévention des risques de toute nature, I'information et l'alerte des populations ainsi que la protection des personnes, des biens et de l'environnement contre les accidents, les sinistres et les catastrophes par la préparation et la mise en œuvre de mesures et de moyens appropriés relevant de l'État, des collectivités territoriales et des autres personnes publiques ou privées » (2). Au-delà des missions traditionnelles, I'actuelle législation prend acte de trois défis pour la politique de sécurité civile: la diversification des risques, des missions et des acteurs.

La sécurité civile s'est organisée, depuis longtemps, pour répondre aux risques collectifs territorialisés. Aujourd'hui, la loi constate « la pluralité des risques pesant sur la population d'une société moderne, les conséquences plus lourdes des phénomènes naturels, la vulnérabilité aux risques technologiques et aux effets de la malveillance » (3). La concentration urbaine de populations, d'activités, de réseaux urbains ou de voies de communication accroît la somme d'enjeux accumulés dans un espace restreint. L'occurrence d'un risque, même situé géographiquement (par exemple les tempêtes Lothar en 1999 et Klaus en 2009), entraîne, dans les espaces urbains denses, des dangers difficilement localisables. La densification urbaine s'amplifiant, les risques s'accumulent, se complètent par effet de domi- no et s'étendent à travers les réseaux. Dans les années 1990, les gestionnaires publics des risques appréhendent les accidents, d'origine naturelle ou industrielle, comme des menaces extérieures (Gilbert, 1992). Leur attention s'oriente aujourd'hui sur les dynamiques internes d'émergence et de propagation de risques dans les systèmes urbains.

Pour y répondre, la loi préconise une gestion globale de la sauvegarde et des secours (4). Elle encourage la coopération surtout la coordination - entre les acteurs publics (les services d'État de protection civile dans les préfectures, les services départementaux d'incendie et de secours, les communes ainsi que leurs intercommunalités) et les acteurs privés, notamment les habitants, les bénévoles, les entreprises et les opérateurs de service. Tous doivent concourir à la continuité du système urbain en cas de risque. Le maintien des services prioritaires (eau, énergie, communication), même en mode dégradé (5), est au cœur des objectifs de la loi de modernisation de la sécurité civile de 2004. Quel que soit le dysfonctionnement entraîné par I'occurrence d'un risque (saturation, arrêt de la production, de I'approvisionnement, de la distribution), la loi considère l'arrêt des services en réseau (6) - même momentané - comme une rupture du fonctionnement urbain.

Les collectivités locales sont directement concernées. La loi de 2004 préconise un plan communal de sauvegarde. Outil phare de la modernisation affichée, il s'agit d'un document de 
planification opérationnelle et d'organisation de la protection civile, régi selon une logique de commandement hiérarchique propre à la gestion de crise.

En s'inspirant des démarches de la sociologie des techniques (Dupuy, 1978; Latour, 1992), le plan communal de sauvegarde peut-il être envisagé comme un « dispositif technique à vocation générique porteur $d^{\prime}$ une conception concrète du rapport politique/société et soutenu par une conception de la régulation » (Lascoumes et Le Galès, 2005, p. 14)? L'étude des conditions d'utilisation du plan communal de sauvegarde, en tant qu'instrument d'action publique, révèle, au-delà des attendus techniques et opérationnels, des rapports de force institutionnels, politiques et territoriaux.

Deux séries d'arguments appuient la démonstration. D'une part, la mise en place du plan communal de sauvegarde permet une redistribution des missions de sécurité civile entre les acteurs publics de la gestion des risques. Certaines intercommunalités se saisissent des nouveaux enjeux pour se positionner dans la gouvernance locale. D'autre part, l'étude des conséquences de l'application du plan communal de sauvegarde donne un cadre d'analyse à la construction politique d'une ville-système dans laquelle "le risque, c'est l'interruption du processus métabolique, le blocage des échanges de flux, la panne urbaine » (Lavigne, 1988, p. 13).

Les deux idées sont illustrées par deux sources principales d'informations. Les résultats d'une étude sociologique, menée sur les conditions de mise en œuvre du plan communal de sauvegarde, sont issus du traitement statistique d'un questionnaire envoyé, en 2008, à un échantillon de 40 communes et de 35 intercommunalités. D'autres exemples sont issus d'entretiens semi-directifs, menés entre 2006 et 2008, dans les services administratifs en charge des risques collectifs des agglomérations de Nantes, de Lyon et du Havre. Sans entrer dans une logique comparatiste, la démonstration s'appuie sur les justifications décisionnelles, produites par les agents administratifs et les élus locaux en charge des risques, dans les communes et leurs intercommunalités.

\section{LE PLAN COMMUNAL DE SAUVEGARDE, UN OUTIL TECHNIQUE ET POLITIQUE}

La loi de modernisation de la sécurité civile de 2004 impose aux communes identifiées " à risque majeur » (7) un délai de trois ans pour refonder leur plan communal de sauvegarde
(PCS). Mis en œuvre sous la responsabilité du maire, le PCS peut néanmoins être réalisé par les intercommunalités (8). En 2008, 40\% des communes concernées par I'obligation l'ont réalisé (Gralepois, 2008a). L'enquête par questionnaire menée sur les PCS cherche à comprendre quels obstacles organisationnels, institutionnels ou territoriaux rencontrent les communes pour s'engager si faiblement dans la démarche. Elle étudie aussi dans quel cas et sous quelles conditions certaines communes apparaissent comme pionnières (9).

II ressort que $90 \%$ des services en charge du risque dans les communes considèrent que les intercommunalités peuvent participer à la gestion des risques. Les exécutifs politiques municipaux sont tout autant parties prenantes. Cette position va à l'encontre de l'idée reçue de " pré carré communal » des politiques de sécurité. Par contre, les intercommunalités s'avèrent réticentes à s'impliquer. Seuls $31 \%$ des services en charge du risque dans les agglomérations acceptent l'idée d'une participation significative.

Pourtant, certaines intercommunalités, telles que celles de Nantes, de Lyon et du Havre, se saisissent rapidement de la réalisation du PCS, pour le compte de leurs communes, comme d'une ressource pour gérer, non seulement les risques majeurs, mais aussi les incidents urbains (10). Quels arguments justifient I'utilisation, au niveau intercommunal, d'outils de sécurité civile, destinés à la gestion de crise, pour des événements récurrents? Que cherchent les services administratifs intercommunaux en travaillant à proximité des pouvoirs de police des maires?

À la lecture des résultats du questionnaire et des entretiens de terrain, les deux arguments incitant les communes à mettre en œuvre régulièrement le PCS sont: I'actualisation d'information et l'expérience de collaboration. Les deux arguments, ancrés dans une logique d'opérationnalité, révèlent un enjeu institutionnel pour l'intercommunalité. Le PCS est plus qu'un outil technique: c'est un moyen de positionnement des intercommunalités face à la disparition des prérogatives " risques » au sein des services communaux de sécurité civile.

\section{L'incident urbain comme moyen de mise à jour des données}

Le plan communal de sauvegarde est un document de format papier ou informatique. II compile la liste des principaux dangers menaçant la commune, puis une série de fiches-actions, 


\section{Une prise en compte des risques collectifs dans la sécurité civile \\ Article 3 du décret du 13 septembre 2005 relatif au plan communal de sauvegarde}

I.- Le plan communal de sauvegarde est adapté aux moyens dont la commune dispose. Il comprend :

a) le document d'information communal sur les risques majeurs [...];

b) le diagnostic des risques et des vulnérabilités locales;

c) I'organisation assurant la protection et le soutien de la population qui précise les dispositions internes prises par la commune afin d'être en mesure à tout moment d'alerter et d'informer la population et de recevoir une alerte émanant des autorités. Ces dispositions comprennent notamment un annuaire opérationnel et un règlement d'emploi des différents moyens d'alerte susceptibles d'être mis en œuvre;

d) les modalités de mise en œuvre de la réserve communale de sécurité civile [...].

II.- Le plan communal est éventuellement complété par :

a) l'organisation du poste de commandement communal mis en place par le maire en cas de nécessité;

b) les actions devant être réalisées par les services techniques et administratifs communaux;

c) le cas échéant, la désignation de l'adjoint au maire ou du conseiller municipal chargé des questions de sécurité civile;

d) l'inventaire des moyens propres de la commune, ou pouvant être fournis par des personnes privées implantées sur le territoire communal. Cet inventaire comprend notamment les moyens de transport, d'hébergement et de ravitaillement de la population. Ce dispositif peut être complété par l'inventaire des moyens susceptibles d'être mis à disposition par l'établissement intercommunal dont la commune est membre;

e) les mesures spécifiques devant être prises pour faire face aux conséquences prévisibles sur le territoire de la commune des risques recensés;

f) les modalités d'exercice permettant de tester le plan communal de sauvegarde et de formation des acteurs;

g) le recensement des dispositions déjà prises en matière de sécurité civile par toute personne publique ou privée implantée sur le territoire de la commune;

h) les modalités de prise en compte des personnes qui se mettent bénévolement à la disposition des sinistrés;

i) les dispositions assurant la continuité de la vie quotidienne jusqu'au retour à la normale.

c'est-à-dire de schémas d'organisation (personnes ressources, matériel logistique, équipements sensibles, etc.). Le PCS inventorie I'ensemble des moyens - publics et privés - potentiellement nécessaires: liste des hébergements, numéro des médecins, lieux de stockage alimentaire, etc. (voir encadré).

De manière unanime dans les collectivités et les services d'État, l'opérationnalité du PCS tient à sa capacité à fournir des informations précises en cas de crise, par exemple des contacts téléphoniques corrects ou des quantités de produits industriels. Le besoin d'opérationnalité constant justifierait alors l'utilisation du PCS pour tout type d'événement survenant dans I'agglomération.

En 2006, les intercommunalités de Nantes, de Lyon et du Havre sont pionnières dans les domaines de gestion des risques. Se saisissant de la loi sur la sécurité civile de 2004, elles font la démonstration de leur rôle d'accompagnement dans la réalisation des PCS, voire défendent la réalisation d'un plan intercommunal de sauvegarde. Dans les trois agglomérations, les acteurs intercommunaux du risque proposent d'utili- ser régulièrement le PCS, c'est-à-dire en tant qu'outil de gestion de crise, pour tout type d'incident. L'objectif affiché est de donner un moyen de vérifier constamment l'exactitude des données.

La communauté urbaine de Lyon possède une mission dédiée au risque majeur dans le service d'écologie urbaine. Le chargé de mission sur les risques majeurs explique pourquoi le PCS doit aussi servir à gérer les accidents de la route.

"Ce matin, on a eu un accident d'autocar, comme ça nous est arrivé déjà il y a trois ans avec un bus de touristes allemands. Même si ce n'est pas un accident majeur, il y a tout de même des personnes tuées. Et pourtant quand ça arrive, là il y a vraiment besoin d'une organisation considérable. (...) Comme ce n'est pas un risque majeur, on n'a pas l'idée de s'y préparer. D'accord, mais il faut être souple sur les définitions et sortir de l'idée que la prévention et la gestion des risques, c'est lié aux risques majeurs. » (Chargé de mission sur les risques majeurs, mission écologie urbaine, Grand Lyon, 14 avril 2006). 
L'idée développée dénonce la séparation entre les procédures de traitement routinier des accidents quotidiens, qui sont déconnectées des méthodes spécifiques de gestion de crise. Chaque accident serait une somme d'informations pratiques à réactualiser, c'est-à-dire à mettre à jour, dans les services de secours (pompiers, police et SAMU). Le chargé de mission "risque » de la communauté urbaine de Lyon semble regretter une perte de connaissances. En somme, il soutient qu'un PCS peut se déployer pour des événements urbains, afin de disposer d'un document régulièrement ajusté par l'ensemble des interventions sur les incidents urbains.

Cette définition est partagée chez les gestionnaires du risque des deux autres agglomérations. Elle s'appuie, d'une part, sur la lecture des guides réalisés par la direction de la défense et de la sécurité civiles (11). Les chargés de mission déduisent que le PCS est aussi un instrument de régulation des incidents perturbant occasionnellement les échanges et les services dans les communes (enneigement de voirie, chute d'équipement sur un chantier, rupture des communications téléphoniques, fuite de canalisation de gaz, incendie, etc.). L'argumentation démontre, d'autre part, qu'un simple incident, s'il n'est pas géré à temps, avec des informations fiables, peut devenir une situation de crise. Tout particulièrement, le dysfonctionnement dans les réseaux urbains, dont la connaissance est parfois partielle chez les gestionnaires publics (notamment pour les anciennes délégations de service), est décrit comme un espace de déstabilisation potentielle de tout le fonctionnement urbain. Cette position conçoit un système urbain intimement lié à la circulation des services en réseau.

\section{La coopération par le vécu de la crise}

L'argument corollaire de l'utilisation régulière du PCS pour les incidents est de créer " une culture du risque ». Les acteurs de la gestion des risques revendiquent ainsi le développement d'un apprentissage collectif des réflexes de sécurité civile. En continuité de l'argument d'actualisation itérative, ils expliquent que les procédures de gestion de crise sont peu testées et, heureusement, peu mises en œuvre. Cette spécificité pose une problématique en termes d'expérience, d'échange et de coordination des procédures et des savoir-faire. Ainsi, I'utilisation récurrente du PCS pour les incidents urbains proposerait un entraînement de coopération entre les différents acteurs de la gestion de crise. Même si les incidents sont d'une ampleur temporelle, spatiale et institutionnelle non comparable à l'occurrence d'une catas- trophe, les acteurs du risque arguent d'une mise en pratique sur site et en temps réel.

Lorsque l'on interroge les acteurs locaux sur la possibilité de « banalisation » du risque majeur, ils démentent unanimement. L'adjoint au maire de Neuville-sur-Saône, dans l'agglomération lyonnaise, soutient la démarche de coopération intercommunale, mais souligne également les bénéfices induits dans l'organigramme municipal. II explique:

"Ce qu'on a voulu à Neuville, c'est que l'ensemble des adjoints et des services techniques aient un document, surtout une culture du risque et des réflexes, pour tous les événements, pas seulement pour les risques majeurs. Les événements sont vécus au quotidien, je veux dire plus ou moins tous les mois. » (Adjoint au maire à I'urbanisme, à I'environnement et aux risques, Ville de Neuville-sur-Saône, 6 novembre 2006).

La potentielle banalisation des incidents lui paraît amplement compensée par les gains acquis lors des tests du PCS. Les gains sont formulés en termes de synchronisation des tâches, de visualisation des modalités d'intervention, de compréhension des cultures professionnelles et institutionnelles.

Dans le cas de Neuville-sur-Saône, la position de l'adjoint au maire n'est pas représentative de celle de l'ensemble des élus locaux, rarement proactifs en matière de politique de prévention et de gestion des risques, mais plutôt de celle - assez spécifique - des élus-techniciens (12). Cette prise de position est plutôt partagée par les agents administratifs des services techniques qui revendiquent l'expérience sensible de l'accident, voire de la crise, comme vecteur essentiel d'apprentissage de la coopération (Coanus et al., 2004).

C'est ce qu'illustre l'extrait d'entretien d'un directeur général aux réseaux urbains de la communauté d'agglomération de la région havraise. L'agglomération havraise est soumise à différents risques d'inondations (ruissellement, débordement, submersion). Depuis les crues dévastatrices successives de 2003 à 2008, les gestionnaires du risque assurent avoir appris d'euxmêmes et des autres. Le directeur général aux réseaux urbains explique:

"On aura beau essayer de réfléchir en se disant qu'on va s'organiser, monter une organisation sur le papier, etc., il n'y a que l'épreuve du terrain qui permet de penser ces choseslà. Il faut être à 3 heures du matin, les pieds dans la boue, 
avec la population à avertir, l'information à faire circuler, les médias qui insistent pour avoir des infos et faire dire ce qu'on ne veut pas dire... C'est là qu'on comprend ce que $c^{\prime}$ est que la gestion de crise et comment dimensionner l'organisation qui va derrière » (Directeur général adjoint au développement durable et aux services à la personne, communauté d'agglomération de la région havraise, 19 décembre 2006).

Le récit presque charnel de l'expérience présente la crue comme un moment fondateur dans la création d'un club local de gestionnaires des risques qui, depuis, échange les informations et se rencontre régulièrement, notamment autour de formations pédagogiques à la gestion de crise. Mais les deux formations annuelles ne semblent pas contrebalancer l'importance d'un usage régulier du PCS pour entretenir les réflexes de coopération intellectuelle et comportementale.

\section{Le plan communal de sauvegarde comme outil de revendication institutionnelle}

La coopération construite lors de I'utilisation régulière du PCS permettrait de mieux connaître l'organisation des acteurs partenaires en cas de crise, mais aussi l'organisation au sein des services communaux. Dans l'enquête par questionnaire sur les PCS, 90\% des communes interrogées présentent la réalisation des PCS comme l'occasion d'une implication plus forte des services municipaux dans la gestion des risques (Gralepois, 2008a, op. cit.). Précisément, dans les agglomérations à l'étude, les gestionnaires des risques - intercommunaux et municipaux souhaitent faire de la mise en place du PCS un moment de réorganisation des missions communales de sécurité civile.

La loi de modernisation de sécurité civile a été bien accueillie dans ses orientations politiques et ses objectifs opérationnels. Beaucoup d'agents administratifs en charge de la gestion des risques dans les communes y ont vu un moyen de confronter les responsables politiques à ce qu'ils considèrent comme un manque de moyens et d'effectifs. La départementalisation des services d'incendie et de secours de 1996 (13) a laissé un vide dans la politique communale de sécurité civile concernant les missions de connaissance des vulnérabilités et de prévention des risques (Padioleau, 2002). Les tâches de sécurité civile dans les communes sont essentiellement tournées vers la sécurité des établissements recevant du public et la gestion des grands événements collectifs (foires, festivals, etc.). Les agents administratifs espèrent qu'en faisant la promotion de
I'utilisation régulière du PCS, ils vont mettre au grand jour - sur des événements mineurs - le sous-dimensionnement en moyens humains, techniques et financiers. Pour ce faire, les communes aspirent à être aidées dans la démarche de réalisation des PCS.

Certains services en charge de la prévention et de la gestion des risques dans les intercommunalités ont rapidement proposé leur participation sur la base de trois séries d'arguments.

D'une part, les services intercommunaux soulignent leur simple rôle de soutien et d'intermédiaire. Les PCS restent, de toute façon, mis en œuvre sous la responsabilité des maires. Ils se proposent de récolter les informations, d'aider à la construction des documents ou à la concertation entre les services municipaux. Ce premier argument de nature institutionnelle mobilise l'idée d'une administration intercommunale politiquement neutre, mutualisant les intérêts communaux (14).

D'autre part, les services soutenant l'implication des agglomérations dans le PCS soulignent que le rôle des intercommunalités est clairement énoncé, à plusieurs reprises, dans la loi de modernisation de la sécurité civile, notamment en ce qui concerne la possibilité de réalisation d'un plan intercommunal de sauvegarde. Ils rappellent aussi que certains pouvoirs de police du maire ont déjà été transférés. Le code général des collectivités territoriales propose au maire, par dérogation aux dispositions de l'article 2212-2, de transférer une partie de ses pouvoirs de police spéciale (police de l'eau, du stationnement, de l'assainissement, des aires de gens du voyage, etc.) (15) en faveur du président de l'intercommunalité de rattachement. La nature juridique des arguments ne fait pas nécessairement autorité dans les communes qui restent très attentistes face aux consignes encore peu claires du Ministère de l'intérieur et des collectivités locales.

Enfin, les services en charge des risques dans les trois agglomérations étudiées juxtaposent, dans leur rhétorique, l'idée d'une utilisation régulière du PCS - pour les deux raisons d'actualisation et de coopération - et leur compétence d'autorité délégante des services publics en réseaux (16), notamment l'exigence de continuité des activités nécessaires à la vie en collectivité, telles que l'accès à l'eau potable, le ramassage des ordures ménagères ou la fluidité des transports.

En réponse aux exigences d'opérationnalité, les services intercommunaux en charge des risques se proposent de tester 
régulièrement le PCS sur les incidents de réseaux urbains qui relèvent de leur compétence. Au départ, les dirigeants des intercommunalités - élus et hauts fonctionnaires - sont réticents à I'organisation intercommunale des services urbains en mode dégradé. Leur consentement s'établit progressivement depuis les épisodes de canicule de 2003, d'épizootie aviaire de 2004 et de grippe A H1N1 à l'automne 2009 (17).

La participation intercommunale à la gestion de crise, notamment pour la prise en charge des réseaux, est fortement soutenue par les services de sécurité civile en préfecture. Ces derniers avouent leurs difficultés à offrir des solutions au diagnostic des risques ou à la mise en œuvre des outils de sécurité civile. D'un côté, la loi de modernisation de sécurité civile de 2004 prévoit la mise en place de nouvelles définitions de la crise, de nouveaux outils et d'une nouvelle gouvernance. Les services de sécurité civile en préfecture pourraient être piliers et relais des nouveaux enjeux. Mais, d'un autre côté, ils ressentent un affaissement franc de leur effectif et de leur soutien ministériel (matériel, budgétaire et institutionnel) qui, selon les questionnaires et les entretiens, ne leur permet pas d'assumer ce rôle. Ils se sentent moins bien dotés que les autres domaines préfectoraux en charge de sécurité intérieure ou militaire. Dès lors, les services de sécurité civile appuient vivement les initiatives intercommunales qui actualiseraient, déjà, un certain nombre des PCS parmi les centaines de communes des départements.

Bien que la légitimité institutionnelle et politique de l'échelon intercommunal en matière de sécurité civile soit largement contestée (Gralepois, 2008b), le manque d'organisation communale et le désarroi des services d'État laissent le champ libre à une mise sur agenda intercommunal. La prise en charge de I'organisation ou de la réalisation des PCS par les agglomérations, pour le compte de leurs communes, renforce le positionnement de l'intercommunalité dans la gouvernance locale. Dans les trois agglomérations étudiées, l'implication des services intercommunaux de prévention et de gestion des risques dans la mise en place des PCS s'est réalisée dans un climat politique étonnamment unanime. Aucun élu ne diverge. De manière générale, les questions de risques urbains, c'est-à-dire les questions de risques hors catastrophes, font rarement l'objet $d^{\prime}$ un portage politique. À part quelques élus-techniciens passionnés, les débats autour de la mise en place des PCS se déroulent dans une arène administrative restreinte, teintée $d^{\prime}$ un discours technicien et gestionnaire, alors que les enjeux sont nombreux et importants.

\section{L'AGGLOMÉRATION À FLUX TENDUS}

Le choix d'une utilisation récurrente du plan communal de sauvegarde pour tout incident urbain sous-tend, selon nous, une définition systémique de l'espace urbain et du risque. La ville est appréhendée comme un espace de concentration, d'accumulation et d'interaction entre différentes fonctions (production, transport ou habitat). Pour justifier leur portage institutionnel, les chargés de mission « risques » dans les intercommunalités développent l'idée d'un espace urbain défini, non pas sur les limites communales, mais sur l'ensemble des interconnexions, des échanges et des réseaux contenus dans l'agglomération. La question des limites et des périmètres n'est pas abordée théoriquement. L'intercommunalité est bien souvent présentée, de fait, comme pertinente dans sa gestion des flux et des réseaux entre communes.

Le risque principal dans la conception de ce système urbain est le blocage des échanges fonctionnels. L'image de la villesystème, en tant qu'espace à flux tendus, développe une approche désectorisée et systémique des risques collectifs. L'usage régulier du PCS ne constitue pas seulement une étape technique et opérationnelle, supposée neutre, comme un trait d'union entre des objectifs politiques et des résultats. II contribue à affaiblir la différence entre l'incident (l'événement localisable et maîtrisable), la catastrophe (accident majeur non anticipé) et la crise (catastrophe non maîtrisée par les pouvoirs publics). Le risque n'est pas appréhendé selon une source d'origine, ni selon une catégorisation de " grave » ou de « mineur». Tout incident est envisagé comme un potentiel perturbateur du système urbain.

L'approche systémique des risques, étudiée dans les années 1980 et 1990 (Dourlens, 1988; Lavigne, 1988; Chaline et Dubois-Maury, 1994; D’Ercole, 1994; November, 1994), est peu actualisée par les travaux de sociologie politique appliquée à l'étude des territoires urbains, exception faite des travaux de géographie sur les vulnérabilités des métropoles (Pigeon, 2005; Reghezza, 2006). Quelle évolution des représentations de la ville traduit I'application du PCS à tout incident? Plus spécifiquement, quelle représentation urbaine est sous-tendue lorsque la circulation dans les réseaux techniques devient un objectif de sécurité civile?

\section{Les pannes de réseaux comme espace de « vigilance des territoires"}

Les objectifs opérationnels et les instruments d'action publique 
produits par les agents intercommunaux de prévention et de gestion des risques traduisent une approche de la panne dans les réseaux urbains (voirie, canalisations, câblages, etc.) comme une crise potentielle. Même si la rupture de flux se présente, dans un premier temps, comme un incident gérable et localisable, elle est perçue comme un espace de développement potentiel d'un événement catastrophique qui doit être encadré par les outils de sécurité civile.

L'utilisation régulière du PCS met en pratique une forme de " vigilance des territoires ». Par exemple, à Villeurbanne, dans la communauté urbaine de Lyon, le PCS est appliqué pour les fuites de gaz, en prévention de potentielles explosions et incendies. Le PCS est testé, à Nantes, lors des interventions sur les pollutions liées au défaut de raccordement des particuliers dans le réseau d'assainissement.

Contrairement au principe de précaution - c'est-à-dire l'attitude qui proscrit de faire courir des risques à des populations si l'on dispose de connaissances ou de moyens insuffisants -, " la mise en vigilance » suggère davantage une posture de prudence (Roux, 2004). Elle se traduit par une attention soutenue aux signaux faibles de dysfonctionnement. En cela, I'utilisation régulière du PCS, pour des blocages momentanés des réseaux urbains, s'apparente à ce que Jacques Roux appelle "I'opérativité discrète » ou les régimes " décontractés » de la vigilance. Ce sont les attitudes de maintien de veille et de réactivité, dans des situations d'apparente accalmie, pour faire face à de simples dysfonctionnements (Roux, 2006).

La mise en place d'outils de veille et d'information est une illustration probante. Un épisode neigeux d'une dizaine de centimètres, à Nantes, en décembre 2005, un jour de grande affluence autour d'un événement musical, a provoqué la congestion des circulations (routières, piétonnières, mais aussi - par extension - économiques et culturelles). L'enneigement a nécessité la mise en place d'un poste de commandement de crise en préfecture. La municipalité était dépassée par les conséquences de l'événement. Pour les agents intercommunaux en charge des risques, cette situation devait être traitée en interne de l'institution intercommunale, par des outils d'alerte et de gestion des signaux faibles de dysfonctionnement des flux urbains.

Les agents intercommunaux développent alors un outil de gestion des incidents qui permette " un maintien de l'éveil » assurant « une capacité à gérer sans panique » pour « se déta- cher de l'anxiété et de l'angoisse ». Depuis 2001, en appui du PCS, la communauté urbaine de Nantes met en place un plan de « mobilisation des moyens logistiques communautaires en cas de crise ». C'est un instrument informatique de localisation, de coordination et de mutualisation, en temps réel, des ressources techniques, humaines ou professionnelles. L'objectif est de pouvoir fournir un « changement d'échelle en montant une strate » lorsqu'un événement prend la dimension d'un accident ou même d'une crise. Cette approche soutien l'idée qu'une défaillance de circulation d'un réseau (de biens, de services et $d^{\prime}$ 'informations) est une menace pour le fonctionnement du système urbain en entier. Dans ce cadre, il n'y a pas de différence de nature, mais de dimension dans la gestion opérationnelle d'un événement ou d'une crise sur l'espace urbain.

Les formes de mise en vigilance des territoires et des réseaux suggèrent deux implications politiques et sociales. Tout d'abord, quand chaque panne de réseau devient un espace de vérification des données ou d'apprentissage des réflexes collectifs, le concept de flux de services l'emporte sur celui d'alimentation en services. L'usage récurrent d'outil destiné à la sécurité des biens et des personnes semble protéger davantage l'échange en lui-même (la circulation) que son contenu (le service) et son objectif (la production, la distribution, la consommation ou l'évacuation). Or, lorsque la recherche de la ville circulatoire se double d'une attitude de vigilance, "le pouvoir des flux prend le pas sur les flux de pouvoir »(Castells, 1998, p. 575). Cette logique traduit bien la conséquence d'une réflexion systémique de l'espace urbain, c'est-à-dire le renforcement de l'emprise technicienne sur la ville, voire la dépolitisation des problèmes de sécurité des réseaux (Dourlens, 1988).

Ensuite, la vigilance constante peut entraîner une relativisation des risques. À juste titre, Dourlens et Vidal-Naquet (1987) soulignent: «la très grande interdépendance des divers composants du système requiert donc un développement des opérations de contrôle et de surveillance qui ne peuvent se limiter aux seuls points névralgiques mais doivent au contraire s'étendre à l'ensemble du (des) réseaux. Cette extension de la fonction de contrôle et de surveillance, rendue possible par la diffusion des nouvelles technologies de l'information, conduit alors à une banalisation du risque puisque "tout peut être surveillé" » (pp. 402-403). Quand tous les dysfonctionnements des réseaux d'échange urbain deviennent insupportables et inacceptables, la ville s'éloigne de son idéal de sécurité et de lien social, pour ressembler de plus en plus à son image la plus 
négative d'espace de contrôle des comportements sociaux. En cela, la prise en charge systématique des dysfonctionnements urbains permet $d^{\prime}$ anticiper puis de réduire tout espace de déstabilisation sociale et politique.

\section{Régulation urbaine et territoriale}

L'utilisation du PCS ne tend pas à empêcher la survenue d'incidents ou à sanctionner le comportement des acteurs. Elle permet de ne pas déterminer ce qu'est un risque et ce qui ne l'est pas, car elle ne délimite pas de segment de temps, d'espace ou de degré de gravité. L'utilisation régulière du PCS s'appuie sur les incidents urbains, en apprenant de leur survenance - par exemple pour actualiser les données ou former les acteurs - pour les contrôler et éviter l'occurrence de risques globaux.

Le traitement systématique des dysfonctionnements de services urbains par les outils territoriaux de sécurité civile traduit une volonté de "régulation urbaine ». Foucault (2004) différencie la sécurité de la discipline: "La sécurité va essayer d'aménager un milieu en fonction d'événements ou de séries d'événements possibles, séries qu'il va falloir régulariser dans un cadre multivalent et transformable. L'espace propre à la sécurité renvoie donc à une série d'événements possibles, il renvoie au temporel et à l'aléatoire, un temporel et un aléatoire qu'il va falloir inscrire dans un espace donné » (p. 22). Là où la discipline délimite et interdit, la sécurité cherche à prévoir et à intégrer les éléments perturbateurs. Les acteurs publics soutenant ce modèle de régulation des risques, appliqué aux incidents qui déstabilisent les échanges urbains, estiment que le PCS pourrait également être étendu à d'autres " nouveaux » types de risques non majeurs (18), comme les manifestations sociales ou l'établissement de camps de gens du voyage, afin de faire fonctionner les systèmes urbains sans obstacle. En ce sens, les caractéristiques de la politique de régulation urbaine ressemblent pour beaucoup aux objectifs prônés dans l'idée de «ville résiliente».

Le terme de « résilience » est employé dans de nombreux domaines (pour les matériaux par exemple) afin d'exprimer la capacité d'un système à maintenir un état stable, afin de poursuivre son fonctionnement, suite à une perturbation (Hollnagel et al., 2006). Dans une vision territoriale, la résilience est à la fois une fragilité et un endommagement, mais aussi un degré de résistance, c'est-à-dire de capacité à faire face (Reghezza, 2006). L'objectif de I'utilisation régulière du PCS est de permettre un retour à la normale le plus rapide possible, qui n'est pas nécessairement le point d'équilibre de la situation précédente. Cette approche technicienne et gestionnaire présume I'amélioration du fonctionnement des réseaux par l'apprentissage des incidents ou même des crises (Milbert, 2003).

\section{ConClusion}

Les modalités concrètes de mise en place des plans communaux de sauvegarde, initiés par la loi de modernisation de sécurité civile en 2004, révèlent des objectifs opérationnels, des rapports de force institutionnels et une vision politique des territoires.

D'un point de vue technique, la légitimité du PCS suppose une opérationnalité constante des données nécessaires en cas de crise. L'utilisation régulière permettrait de favoriser les interactions entre les échelles temporelle et spatiale, en offrant un outil redimensionnable de planification, de l'incident à l'accident jusqu'à la crise.

L'utilisation du PCS est un outil de revendication institutionnelle. Le manque de moyens des services de sécurité civile des communes et des préfectures offre une fenêtre d'opportunité pour une mise à l'agenda intercommunal. Bien que les démarches des administrations locales présentées semblent isolées, elles s'appuient sur une interprétation des textes de lois de sécurité civile. Les intercommunalités semblent être porteuses de l'idée qu'un risque potentiellement catastrophique se trouve dans toute panne urbaine. Elles diffusent cette idée au sein des cercles institutionnels de « bonnes pratiques » sur les politiques de prévention et de gestion des risques. En soutenant la nécessité de penser les interconnexions de territoires et de réseaux, les agents en charge des risques dans les agglomérations font de I'intercommunalité une échelle de dépassement nécessaire pour gérer les crises. L'intercommunalité apparaît légitimée dans son rôle d'institution d'intégration et de résolution des dysfonctionnements des systèmes de relations supra-communales.

La vision véhiculée par les services intercommunaux de prévention et de gestion des risques traduit une image de "ville-système », qui serait définie dans l'agglomération, en tant qu'espace de circulations à flux tendus, dont le principal dan- 
ger est le blocage, même ponctuel, des échanges fonctionnels. En somme, la mise en place du PCS est un levier d'argumentation pour porter une vision territoriale de la sécurité civile: celle de la régulation des systèmes urbains. Cette vision ne porte pas une réflexion économique et sociale sur le contenu du flux, mais consacre la circulation urbaine comme un préalable au développement urbain des agglomérations.
Mathilde Gralepois est maitre de conférences dans le département d'aménagement de l'École Polytechnique Universitaire à l'Université de Tours. Après avoir soutenu sa thèse en novembre 2008 à I'Université Paris-Est (laboratoire Territoires, Techniques et Sociétés LATTS), elle poursuit ses recherches sur la prise en compte des risques dans l'aménagement des territoires, les approches territoriales de la sécurité civile et le rôle des intercommunalités dans la gouvernance locale au sein du laboratoire CITERES (Centre Interdisciplinaire Cités, Territoires, Environnement et Sociétés). Mathilde.Gralepois@univ-tours.fr

\section{NOTES}

(1) Loi n²004-811 du 13 août 2004 de modernisation de la sécurité civile.

(2) En ce sens, la politique de sécurité civile se distingue de la sécurité intérieure, qui concerne le maintien de l'ordre public et la lutte contre la délinquance.

(3) Préambule aux orientations de la politique de sécurité civile, annexe de la loi de modernisation de la sécurité civile du 13 août 2004.

(4) La sauvegarde est l'ensemble des tâches destinées à assurer la mise à l'abri, I'hébergement des populations, la fluidité des voiries pour que les acteurs du « secours » puissent procéder au sauvetage des personnes et à la maîtrise de l'accident.

(5) « Le caractère prioritaire des besoins de la population (...) se détermine en considération, d'une part, des objectifs de préservation de la vie humaine, de la santé publique, de la sécurité des personnes et des biens et, d'autre part, de la continuité des services publics » (Décret n²007-1400 du 28 septembre 2007 relatif à la définition des besoins prioritaires de la population et aux mesures à prendre par les exploitants d'un service destiné au public lors de situations de crise).

(6) La notion de continuité des services publics en cas d'accidents ou de crises, liés à des risques collectifs, ne recouvre pas le débat sur le service minimum qui désigne l'obligation faite aux salariés de certains services publics d'assurer un fonctionnement sommaire en temps de grève sociale et/ou professionnelle.

(7) Deux catégories de communes sont tenues de réaliser leur PCS: d'une part, les communes couvertes par un plan de prévention des risques (PPR), c'est-à-dire un document réglementaire qui délimite les zones exposées aux risques encadrées par des mesures de prévention, de protection et de sauvegarde des personnes (Loi n95-101 du 2 février 1995 relative au renforcement de la protection de l'environnement); d'autre part, les communes concernées par un plan particulier d'intervention (PPI), c'est-à-dire un plan des secours interne aux entreprises industrielles exposées à des accidents dont les conséquences déborderaient largement le cadre de l'usine.

(8) L'intercommunalité est le nom générique donné dans ce texte pour désigner les Établissements Publics de Coopération Intercommunale (EPCI), créés sous leur forme actuelle par la loi n99-586 du 12 juillet 1999 relative au renforcement et à la simplification de la coopération intercommunale, tels que les communautés urbaines et les communautés d'agglomération.

(9) Dans $70 \%$ des cas, dans les communes comme dans les intercommunalités, le questionnaire a été rempli par les services en charge des risques collectifs ou par la direction générale des services. Pour retrouver les résultats de l'enquête et ses méthodes d'échantillonnage, voir la page HAL-SHS en renseignant le nom de l'auteure: http://halshs.archives-ouvertes.fr

(10) L'incident est un dommage imprévu, mais quantifiable et localisable, d'ampleur circonscrite: c'est un " accident mâ̂trisable ». La catastrophe est un accident majeur, dont l'ampleur temporelle et géographique, ainsi que les conséquences sur les personnes, les biens et l'environnement n'ont pas été anticipées. La crise est une catastrophe, potentiellement irréversible, qui n'est ni anticipée, ni maîtrisée. L'incertitude déstabilise les pouvoirs publics et les experts scientifiques qui, dans le temps long, perdent leur autorité.

(11) « Méthode d'élaboration: un PCS pour faire face à quoi? » in: Plan Communal de Sauvegarde: Mémento, Direction de la Défense et de la Sécurité Civiles, sous-direction de la gestion des risques, en partenariat avec I'Institut des Risques Majeurs de Grenoble, novembre 2005.

(12) Dans les trois agglomérations étudiées, les élus (adjoints ou maires) intéressés par les enjeux de la gestion de crise sont souvent des professionnels ou des retraités des métiers de l'industrie, de la chimie, de la pharmacie, etc.

(13) La loi n $96-369$ du 3 mai 1996 relative aux services $d^{\prime}$ incendie et de secours, et son décret d'application nº7-1225 du 26 décembre 1997 relatif à l'organisation des services d'incendie et de secours.

(14) L'idée d'une construction intercommunale, qui aurait une visée strictement fonctionnelle ainsi qu'une composition territoriale homogène et pertinente, a été largement remise en cause depuis les travaux de Baraize et Négrier (2001).

(15) Par contre, aux côtés des pouvoirs de police spéciale, 
tels que ceux cités dans le texte, il existe un pouvoir général de police administrative, inaliénable de la fonction de maire.

(16) Depuis la loi de renforcement et de simplification intercommunale du 12 juillet 1999, les communes participant à un établissement public de coopération intercommunale peuvent déléguer la gestion des services d'eau, d'assainissement, d'éclairage, de voirie et de transport. Ce sont des compétences traditionnellement transférées massivement aux intercommunalités.
(17) Le rôle des risques sanitaires dans la prise en compte des réseaux comme vecteur de propagation des risques, par les acteurs administratifs et politiques, demande une étude en soi (Ali et Keil, 2006).

(18) La nouveauté d'un risque ne réside pas dans son caractère inédit, mais dans le fait qu'il est nouvellement labellisé comme risque par les gestionnaires publics, les médias ou tout type d'acteur social.

\section{BibliograPHIE}

Ali S., KeIL R., 2006, "Global Cities and the Spread of Infectious Disease: The Case of Severe Acute Respiratory Syndrome (SARS) in Toronto, Canada", Urban Studies, vol. 43, pp. 491- 509.

Baraize F., NéGrier E. (sous la direction de), 2001, L'invention politique de l'agglomération, Paris, L'Harmattan

Castells M., 2001 (trad. fr.), "L'espace de flux », In: L'ère de I'Information, Tome 1, La Société en Réseaux (1998), Paris, Fayard, pp. 473-530.

Chaline C., Dubois-Maury J., 1994, La ville et ses dangers, Paris, Masson

Coanus T., Duchêne F., Martinais E., 2004, "Risque, territoire et longue durée: Vers une société du risque? ", Annales de la recherche urbaine, $n^{\circ} 95$, Juin, pp. 19-25.

D’Ercole R., 1994, "Les vulnérabilités des sociétés et des espaces urbanisés », Revue de géographie alpine, tome 82, $\mathrm{n}^{\circ} 4$, pp. 87-96.

Dourlens C., 1988, "Villes, risques et périls », Annales de la recherche urbaine, $\mathrm{n}^{\circ} 40$, pp. 2-10.

DOURLENS C., VIDAL-NAQUet, 1987, « Vers une gestion probabiliste du risque? »In: La société vulnérable. Évaluer et mâ̂triser les risques, Fabiani J-L., Theys J. (dir.), Paris, Presses de l'école normale supérieure

Dupuy G., 1978, Urbanisme et technique. Chronique d'un mariage de raison, Paris, Centre de recherche d'urbanisme

Foucault M., 2004, Sécurité, territoire, population. Cours au Collège de France (1977-1978), Paris, Gallimard

Gilbert C., 1992, Le pouvoir en situation extrême. Catastrophes et politiques, Paris, L'Harmattan

Gilbert C. (dir.), 2006, L'État face aux risques, La Documentation Française, $n^{\circ} 328$.

Gralepois M., 2008 (a), Le plan communal de sauvegarde. Une approche territoriale de la sécurité civile à travers l'enquête des conditions de mise en place dans les communes françaises, 5e Commission Risques Industriels-Transports, Conseil National de la Protection Civile

Gralepois M., 2008 (b), Les risques collectifs dans les agglomé- rations françaises à travers le parcours des agents administratifs locaux, Doctorat en aménagement de l'espace-urbanisme, sous la direction de Bernard Barraqué, Université Paris-Est

Hollnagel E., Woods D., Leveson N., 2006, Resilience engineering. Concepts and precepts, Hampshire, Ashgate

LASCOUMES P., 1990, "Normes juridiques et mise œuvre des politiques publiques ", L'Année Sociologique, vol. 40, pp. 43-51.

Lascoumes P., Le Galès P., 2005, Gouverner par les instruments, Paris, Les presses de Sciences Po

Latour B., 1992, Aramis ou l'amour des techniques, Paris, La Découverte

LAVIGNE J-C., 1988, "Au fil du risque des villes », Annales de la recherche urbaine, $\mathrm{n}^{\circ} 40$, pp.11-16.

MilberT I., 2003, "Vulnérabilité et résilience des métropoles: sont-elles sont si fragiles? », In: Développement durable et aménagement du territoire, Da Cunha A., Ruegg J. (dir.), Lausanne, Presses polytechniques et universitaires romandes, pp. 313-330.

NOVEMBer V., 1994, "Risques naturels et croissance urbaine: réflexion théorique sur la nature et le rôle du risque dans l'espace urbain ", Revue de géographie alpine, tome 82, $\mathrm{n}^{\circ} 4$, pp. 115-123.

Padioleau J-G., 2002, Le réformisme pervers: le cas des sapeurs-pompiers, Paris, PUF

Pigeon P., 2005, Géographie critique des risques, Paris, Economica

REGHEZZA, 2006, Réflexions autour de la vulnérabilité métropolitaine: la métropole Parisienne face au risque de crue centennale, Doctorat en géographie, sous la direction d'Yvette Veyret, Université Paris X

Roux J. (dir.), 2006, Etre vigilant. L'opérativité discrète de la société du risque, St-Étienne, Publications de l'Université de St-Étienne

Roux J., 2004, "La ville par précaution », Annales de la recherche urbaine, $\mathrm{n}^{\circ} 95$, pp. 43-46. 